\title{
New earthworm records from Turkey (Clitellata: Lumbricidae, Megascolecidae)
}

\author{
T. SZEDERJESI ${ }^{1}$ and M. MISIRLIOĞLU ${ }^{2}$ \\ ${ }^{I}$ Tímea Szederjesi, Department of Zoology, Plant Protection Institute, Centre for Agricultural Research, \\ Hungarian Academy of Sciences, H-1525 Budapest, P.O. Box 102, Hungary, e-mail: \\ t.szederjesi@gmail.com \\ ${ }^{2}$ Mete Misırlıoğlu, Department of Biology, Faculty of Science and Letters, Eskişehir Osmangazi \\ University, 26480 Eskişehir,Turkey, e-mail: metem@ogu.edu.tr
}

\begin{abstract}
Elaboration of an earthworm material collected in different parts of Turkey resulted in recording altogether eighteen species and subspecies. The Levantine Dendrobaena orientalis Černosvitov, 1940 was reported for the first time outside the Levant. The new occurrence of the Caucaso-Anatolian D. alpina armeniaca (Rosa, 1893) from Hatay province, Levantine Turkey shows that this species has a much larger distribution area than previously expected.
\end{abstract}

Keywords. Earthworms, Turkey, new records, Dendrobaena alpina armeniaca

\section{INTRODUCTION}

$\mathrm{T}$ he beginning of the organized earthworm researches in Turkey is dated to the end of the 1980s', when the first larger scale expeditions took place. These two collecting trips, led by the Italian zoologist Pietro Omodeo, mainly focused on the northern and the south-western part of the country. As a result, several new species and a new genus were described (Omodeo \& Rota 1989, 1991).

Another milestone in the earthworm researches of Turkey was the comprehensive work of Csuzdi et al. (2006), compiling and critically reviewing all previous earthworm records from the country. Since then, the researches are more or less continuous: seven new species were described from different parts of Turkey in the last decade (Csuzdi et al. 2007, Szederjesi et al. 2014a, 2014b) and also a huge amount of new data were published as a result of the different faunistical studies (Misırlığlu 2008, 2010, 2010, Misırlıoğlu \& Szederjesi 2015).

This present paper summarizes the results of the collecting expeditions to different parts of Turkey, accomplished between the years 2006 and 2016.

\section{MATERIAL AND METHODS}

Earthworms were collected by digging and hand-sorting. The specimens were killed and fixed in $80 \%$ ethanol.

The valid names are given according to the online name database of Csuzdi (2012).

\section{RESULTS}

\section{Family Lumbricidae Rafinesque-Schmaltz, 1815}

\section{Allolobophora chlorotica (Savigny, 1826)}

Enterion chloroticum Savigny, 1826: 182.

Allolobophora chlorotica: Csuzdi et al. 2006: 2.

Material examined. 2 ex., Muğla, Ulaş district, leg. F. Türkmen, 17.01.2015. 


\section{Aporrectodea caliginosa caliginosa (Savigny, 1826)}

Enterion caliginosum Savigny, 1826: 180. Aporrectodea caliginosa (part.): Csuzdi et al. 2006: 4.

Material examined. 12 ex., Adana, Sarıçam district, barren land, $\mathrm{N} 37^{\circ} 01.976^{\prime}$, E035 $24.987^{\prime}$, 179 m, leg. B. Or, 10.09.2015.

\section{Aporrectodea caliginosa trapezoides (Dugès, 1828)}

Lumbricus trapezoides Dugès, 1828: 289.

Aporrectodea caliginosa trapezoides: Misırlığlu \& Szederjesi 2015: 101.

Material examined. 3 ex., Karaman, Doğu Kışla quarter, barren countryside, non-grass area, leg. A. Çınar, 05.12.2009. 11 ex., Konya, Karatay, Taşra, Üzümlü quarter, Kanal street, grassy land, leg. O. Şen, 26.04.2015. 24 ex., Muğla, Milas district, Kafaca village, leg. F. Türkmen, 30.03.2014. 3 ex., Konya, Doğanhisar, Başköy village, shore of Başköy pond, leg. M.A Biçer, 02.11.2012. 5 ex., Muğla, Ulaş district, Beçin, leg. F. Türkmen, 31.03.2014. 23 ex., Eskişehir, Sivrihisar district, Bahçecik, wooded region, village fountain, leg. E. Y1lmaztürk, 22.11.2015. 2 ex., Aydın, Kuşadası district, Davutlar area, home garden, tomato plantation, leg. D. Çankaya, 18.08.2013. 13 ex., Afyon, Emirdağ district,Yusufağaç, home garden, among plant roots, leg. B. Şengül, 21.04.2014. 2 ex., Ankara, Güdül district, Kocakaya Mt, Tekke fountain, grassy area with oak trees, leg. A. Şen, 28.11.2009. 9 ex., Karaman, Kisecik, fields surrounding the canal, leg. A. Çınar, 05.12.2009. 1 ex., Konya, Ilgın, pasture, leg. M.A. Biçer, 04.11.2012. 1 ex., Muğla, Ulaş district, leg. F. Türkmen, 17.01.2015. 5 ex., Kocaeli, Gebze district, Istasyon quarter, Darıca turnout, barren land, leg. A. Çinar, 06.12.2009. 1 ex., Muğla, Milas district, Karağaç, leg. F. Türkmen, 04.10.2014. 2 ex., Kırklareli, Lüleburgaz district, Yenibedir, $80 \mathrm{~m}$, leg. O. Mol, M. Karavuş, O. Şen. 5 ex., Adana, Karaisalı district, Gökkuyu Village, roadside, barren land, $\mathrm{N} 37^{\circ} 04.222^{\prime}, \mathrm{E} 035^{\circ} 05.727^{\prime}, 180 \mathrm{~m}$, leg. B. Or,
10.09.2015. 1 ex., Muğla, Ulaş district, leg. F. Türkmen, 17.01.2015. 4 ex., Muğla, Milas district, Kafaca, leg. F. Türkmen, 06.10.2014. 1 ex., Edirne, Kırkpınar district, leg. O. Mol, M. Karavuş, O. Şen, 18.01.2014. 1 ex., Sakarya, Merkez, city center, leg. A.M. Bozkurt, H. Sayın, 11.11. 2015. 3 ex., Edirne, Sarayakpınar, leg. O. Mol, M. Karavuş, O. Şen, 18.01.2014. 6 ex., Muğla, Milas district, Kafaca, leg. F. Türkmen, 04.10.2014. 2 ex., Bilecik, Merkez, Osmangazi quarter, Beyond TOKI Houses, under blackberry, leg. Ö. Perk, 11.12.2016. 2 ex., Eskisehir, Tepebaş1 district, Kumlubel quarter, home garden, leg. B. Şengül, 26.03.2014.

\section{Aporrectodea jassyensis (Michaelsen, 1891)}

Allolobophora jassyensis Michaelsen, 1891: 15. Aporrectodea jassyensis: Csuzdi et al. 2006: 6.

Material examined. 2 ex., Muğla, Ulaş district, leg. F. Türkmen, 17.01.2015. 3 ex., Muğla, Çamköy, leg. F. Türkmen, 23.01.2015.

\section{Aporrectodea rosea (Savigny, 1826)}

Enterion roseum Savigny, 1826: 182.

Aporrectodea rosea: Csuzdi et al. 2006: 6.

Material examined. 7 ex., Konya, Karatay, Taşra, Üzümlü quarter, Kanal street, grassy land, leg. O. Şen, 26.04.2015. 1 ex., Artvin, Murgul district, near the State Hospital, under snow, barren land, leg. Ş. Oktay, 13.12.2016. 11 ex., Afyon, Emirdağ district,Yusufağaç, home garden, among plant roots, leg. B. Şengül, 21.04.2014. 8 ex., Muğla, Ulaș district, leg. F. Türkmen, 17. 01.2015. 3 ex., Muğla, Milas district, Kafaca, leg. F. Türkmen, 04.10.2014.

\section{Dendrobaena alpina armeniaca (Rosa, 1893)}

(Figure 1)

Allolobophora alpina v. armeniaca Rosa, 1893: 431. Dendrobaena alpina armeniaca: Csuzdi et al. 2006: 8.

Material examined. 1 ex., Eskişehir, Beşik Deresi, edge of the stream, leg. K. Kayal,, 
07.05.2013. 1 ex., Hatay, Islahiye district, 1774 $\mathrm{m}$, around Pinus nigra, leg. K. Özgişi, 01. 06.2015 .

External characters. Length 36-59, width 3$4.5 \mathrm{~mm}$. Number of segments 115-131. Pigmentation slight pinkish. Prostomium epilobous $1 / 2$ closed. First dorsal pore at 5/6. Setae distantly standing. Setal arrangement behind clitellum: $a a: a b: b c: c d: d d=2.6: 1.2: 1.3: 1: 2.9$. Male pores on segment 15 , surrounded by a glandular crescent Nephridial pores irregularly alternate between setal line $b$ and above $d$. Clitellum on segments 27-33, 1/n34. Tubercula pubertatis on segments 30-32.

Internal characters. Dissepiments 6/7-12/13 slightly thickened, 13/14-14/15 thickened. Testes and funnels paired in segments 10-11, covered by perioesophageal testis sac in segment 10 and 11. Three pairs of seminal vesicles in $9,11,12$. Two pairs of spermathecae in 9/10,10/11 with external openings near the mid-dorsal line. Calciferous glands in segment 11-12. The last pair of hearts in 10. Nephridial bladders octaedra-type, rarely sausage-shaped. Crop in segments 15-16, and gizzard in segments 17-18. Typhlosolis lamelliform. The cross-section of the longitudinal muscle layer is of pinnate type.
Remark. D. alpina armeniaca is distributed on the northern part of the county. This is its first record from Hatay, the Levantine part of Turkey.

\section{Dendrobaena hortensis (Michaelsen, 1890)}

Allolobophora subrubicunda var. hortensis Michaelsen, 1890: 15.

Dendrobaena hortensis: Csuzdi et al. 2006: 10.

Material examined. 9 ex., Ankara, Güdül district, Kocakaya Mt, Tekke fountain, grassy area with oak trees, leg. A. Şen, 28.11.2009. 12 ex., Kocaeli, Gebze district, Istasyon quarter, Darıca turnout, barren land, leg. A. Çinar, 06.12.2009. 3 ex., Samsun, Arakum, DSI street, asphalt road edge, grassy and bushy area, leg. Y. Y1lan, 22.11.2009.

\section{Dendrobaena orientalis Černosvitov, 1940}

(Figure 2)

Dendrobaena orientalis Černosvitov, 1940: 444. Csuzdi et al. 2006: 11.

Material examined. 1 ex., Konya, Karatay, Taşra, Üzümlü quarter, Kanal street, grassy land, leg. O. Şen, 26.04.2015. 1 ex., Hatay, Islahiye district, $1774 \mathrm{~m}$, around Pinus nigra, leg. K. Özgişi, 01.06.2015.

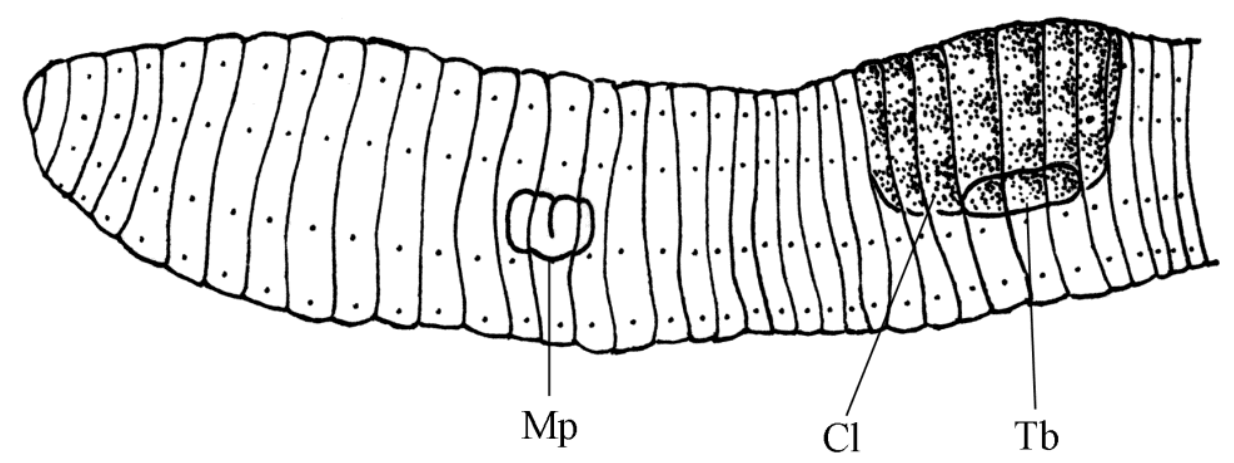

Figure 1. Ventrolateral view of Dendrobaena alpina armeniaca (Rosa, 1893). $\mathrm{Cl}=$ clitellum, $\mathrm{Tb}=$ tubercles, $\mathrm{Mp}=$ male pore. 
External characters. Length 24-41, width 2.5$3 \mathrm{~mm}$. Number of segments 93-99. Pigmentation red-violet. Prostomium tanylobous. First dorsal pore at 5/6. Setae distantly standing. Setal arrangement behind clitellum: $a a: a b: b c: c d: d d=$ 1.8:1.1:1.6:1:1.9. Male pores on segment 15, surrounded by a glandular crescent, protruding into the neighbouring segments. Nephridial pores irregularly alternate between setal line $b$ and above $d$. Clitellum on segments 23, 24-33. Tubercula pubertatis on segments 30-32. Glandular tumescence on 10, $15 a b$.

Internal characters. Dissepiments 6/7-12/13 slightly thickened. Testes and funnels paired in segments 10-11, covered by perioesophageal testis sac in segment 10 and 11 . Three pairs of seminal vesicles in $9,11,12$. Two pairs of spermahecae in 9/10, 10/11 with external openings in setal line $c$. Calciferous glands in segment 11-12. The last pair of hearts in 11. Nephridial bladders octaedra-type. Crop in segments 15-16, and gizzard in segments 17-18. Typhlosolis bilobate. The cross-section of the longitudinal muscle layer is of pinnate type.

Remark. This is a Levantine species, recorded in Turkey only from Hatay (Levantine part of Turkey). This is the first record of $D$. orientalis from the Anatolian part.

\section{Dendrobaena pentheri (Rosa, 1905)}

Allolobophora (Notogama) pentheri Rosa, 1905: 6. Dendrobaena pentheri: Csuzdi et al. 2006: 12.

Material examined. 2 ex., Konya, Karatay, Taşra, Üzümlü quarter, Kanal street, grassy land, leg. O. Şen, 26.04.2015. 1 ex., Mersin, Aslanköy, 1818 m, leg. K. Özgişi, 30.05.2015.

\section{Dendrobaena veneta (Rosa, 1886)}

Allolobophora veneta Rosa, 1886: 674.

Dendrobaena veneta veneta: Csuzdi et al. 2006: 15.

Material examined. 1 ex., Konya, Doğanhisar, Başköy village, shore of Başköy pond, leg. M.A Biçer, 02.11.2012. 1 ex., Eskişehir, Beşik Deresi, edge of the stream, leg. K. Kayal1, 07.05.2013. 1 ex., Artvin, Murgul district, near the State Hospital, under snow, barren land, leg. Ş. Oktay, 13.12.2016. 10 ex., Konya, Ilgin, pasture, leg. M.A. Biçer, 04.11.2012. 1 ex., Eskişehir, Seyitgazi district, home garden with ornamental plants, leg. Y. Dikici, 26.09.2015. 1 ex., Muğla, Ulaş district, leg. F. Türkmen, 17.01.2015. 2 ex., Eskisehir, Tepebaş1 district, Kumlubel quarter, home garden, leg. B. Şengül, 26.03.2014.

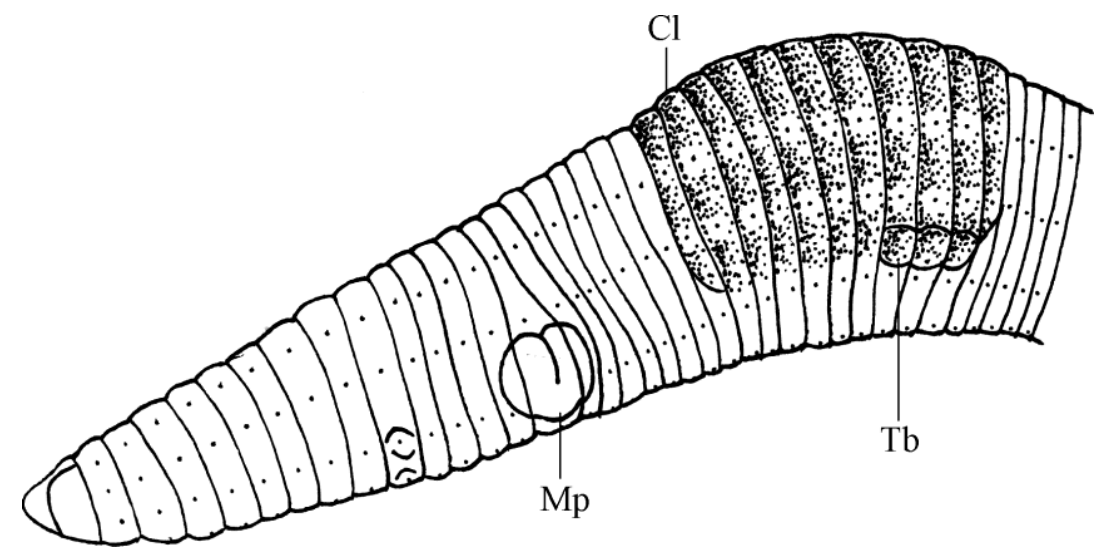

Figure 2. Ventrolateral view of Dendrobaena orientalis $\breve{C}$ ernosvitov, $1940 . \mathrm{Cl}=$ clitellum, $\mathrm{Tb}=$ tubercles, $\mathrm{Mp}=$ male pore 


\section{Eisenia fetida (Savigny, 1826)}

Enterion fetidum Savigny, 1826: 182.

Eisenia fetida: Csuzdi et al. 2006: 18.

Material examined. 3 ex., Tekirdağ, Karacak1lavuz quarter, pasture, leg. E. Uzun, 16.12.2016. 5 ex., Adana, Karaisalı district, Gökkuyu Village, roadside, barren land, $\mathrm{N} 37^{\circ} 04.222^{\prime}, \mathrm{E} 035^{\circ} 05.727^{\prime}$, $180 \mathrm{~m}$, leg. B. Or, 10.09.2015.

\section{Eiseniella tetraedra (Savigny, 1826)}

Enterion tetraedrum Savigny, 1826: 184.

Eiseniella tetraedra: Csuzdi et al. 2006: 19.

Material examined. 1 ex., Ankara, Güdül district, Kocakaya Mt, Tekke fountain, grassy area with oak trees, leg. A. Şen, 28.11.2009. 1 ex., Muğla, Milas district, Karağaç, leg. F. Türkmen, 04.10.2014.

\section{Healyella syriaca (Rosa, 1893)}

Allolobophora syriaca Rosa, 1893: 461.

Healyella syriaca: Csuzdi et al. 2006: 22.

Material examined. 10 ex., Adana, Misis district, under trees, N36 $57.427^{\prime}$, E035 $37.495^{\prime}$, 18 m, leg. B. Or, 10.09.2015. 2 ex., Amasya, Merzifon district, $\mathrm{S}$ of Gökçebağ village,grassy area, leg. Y. Y1lan, 25.11.2009. 2 ex., Amasya, Merzifon district, Tavşan Mt. slopes, Kürt river, leg. Y. Yılan, 29.11.2009. 1 ex., Amasya, Merzifon district, Tavşan Mt. eastern slopes, edge of the Yakacık village road, leg. Y. Y1lan, 29.11. 2009.

\section{Lumbricus rubellus Hoffmeister, 1843}

Lumbricus rubellus Hoffmeister, 1843: 187. Csuzdi et al. 2006: 23.

Material examined. 3 ex., Edirne, Kırkpınar district, leg. O. Mol, M. Karavuş, O. Şen, 18.01. 2014. 1 ex., İstanbul, Yakacık, Sabiha Gökçen Airport surroundings, leg. M. Buldak, 06.10.2006.

\section{Octodrilus complanatus (Dugès, 1828)}

Lumbricus complanatus Dugès, 1828: 289.

Octodrilus complanatus: Csuzdi et al. 2006: 24.

Material examined. 1 ex., Bursa, Yenişehir district, Söylemiş, Karaca stream bank, grassy area, leg. I. Sar1, 19.04.13.

\section{Octodrilus transpadanus (Rosa, 1884)}

Allolobophora transpadana Rosa, 1884: 45.

Octodrilus transpadanus: Csuzdi et al. 2006: 24.

Material examined. 1 ex., Edirne, Kirkpınar district, leg. O. Mol, M. Karavuş, O. Şen, 18.01. 2014. 2 ex., Bilecik, Merkez, Osmangazi quarter, Beyond TOKI Houses, under blackberry, leg. Ö. Perk, 11.12.2016.

\section{Octolasion lacteum (Örley, 1881)}

Lumbricus terrestris var. lacteus Örley, 1881: 584. Octolasion lacteum: Csuzdi et al. 2006: 24.

Material examined. 1 ex., Muğla, Ulaş district, leg. F. Türkmen, 17.01.2015.

\section{Family Megascolecidae Rosa, 1891}

$$
\text { Amynthas corticis (Kinberg, 1867) }
$$

Perichaeta corticis Kinberg, 1867: 102.

Amynthas corticis: Misırlığlu 2012: 104.

Material examined. 1 ex., Adapazarı, Sapanca district, Uzunkum, leg. S. Gençay, 04.12.2011.

\section{DISCUSSION}

The present study resulted in recording altogether 18 species and subspecies of which 17 belong to the family Lumbricidae. Most of the recorded species are peregrine and / or widely distributed, eg. the Trans-Aegean Aporrectodea jassyensis (Michaelsen, 1891) or the Circum-Mediterranean Octodrilus complanatus (Dugès, 1828). 
The Levantine Dendrobaena orientalis Černosvitov, 1940 was first reported outside the Levant, which suggest that the distribution area of this species is probably much larger than previously expected. The situation is similar in case of D. alpina armeniaca (Rosa, 1893) - this species was known mostly from the northern part of the country, but the new record from Hatay shows that the area of this species crosses the ranges of the Taurus Mts to south.

\section{REFERENCES}

CsUzDI, Cs. (2012): Earthworm species, a searchable database. Opuscula Zoologica (Budapest), 43(1): 97-99.

CsuZDi, Cs., ZiCsI, A. \& MisirlioĞLU, M. (2006): An annotated checklist of the earthworm fauna of Turkey (Oligochaeta: Lumbricidae). Zootaxa, 1175: $1-29$.

Csuzdi, Cs., PAvlíčeK, T. \& MisirlioĞLU, M. (2007): Earthworms (Oligochaeta: Lumbricidae, Criodrilidae and Acanthodrilidae) of Hatay Province, Turkey, with description of three new lumbricids. Acta Zoologica Academiae Scientiarum Hungaricae, 53(4): 347-361.

DUGÈs, A. (1828): Recherche sur la circulation, la respiration, et la reproduction des Annélides abranches. Annales des Sciences Naturelles Paris, 15: 284-336.

HOFFMESITER, W. (1843): Beitrag zur Kenntnis deutscher Landanneliden. Archiv für Naturgeschichte, 91: 183-198.

KINBERG, J.G.H. (1867): Annulata nova. Öfversigt af Kongliga Vetenskaps-Akademiens Förhandlingar, Stockholm, 23: 97-103, 356-357.

MichAELSEN, W. (1890): Die Lumbriciden Norddeutschlands. Jahrbuch der Hamburgischen Wissenschaftlichen Anstalten, 7: 1-19.

Michaelsen, W. (1891): Oligochaeten des Naturhistorischen Museums in Hamburg IV. Jahrbuch der Hamburgischen Wissenschaftlichen Anstalten, 8: $1-42$.

MisiRLIOĞLU, M. (2008): Some Earthworm Records from Anatolia (Oligochaeta, Lumbricidae). Turkish Journal of Zoology, 32: 469-471.
MisiRLIOĞLU, M. (2010): Distribution of endemic earthworm species in Turkey (Oligochaeta: Lumbricidae). Zoology in the Middle East, 51(s2): 83-87. doi: 10.1080/09397140.2010.10638461

MisIRLIOĞLU, M. (2012): Distribution of earthworms belonging to families Acanthodrilidae, Criodrilidae, and Megascolecidae in Turkey. Zoology in the Middle East, 58(s4): 103-106. doi: 10.1080/09397140.2012.10648990

MisiRlioĞLU, M. \& SzEDERJESI, T. (2015): Contributions to the earthworm fauna (Oligochaeta) of Turkey. Megadrilogica, 18(6): 99-102.

OMOdeO, P. \& RotA, E. (1989): Earthworms of Turkey. Bolletino di Zoologia, 56: 167-199. doi: $10.1080 / 11250008909355639$

OMODEO, P. \& RotA, E. (1991): Earthworms of Turkey. II. Bolletino di Zoologia, 58: 171-181. doi: $\underline{10.1080 / 11250009109355749}$

ÖRLEY, L. (1881): A magyarországi Oligochaeták faunája. I. Terricolae. Mathematikai és Természettudományi Közlemények, 16: 562-611.

Rosa, D. (1886): Note sui lombrici del Veneto. Atti del Reale Istituto Veneto di Scienze, 4: 673-687.

RosA, D. (1893): Revisione dei Lumbricidi. Memoire della Reale Academia delle Scienze di Torino, 43: 399-477.

RosA, D. (1905): Terricolen. In: Ergebnisse einer Naturwissenschaftlichen Reise zum Erdschias Dag. Annalen des K.K. Naturhistorischen Hofmuseums Wien, 20: 104-106.

SAVignY, J.C. (1826): Analyse des Travaux de l'Académie royale des Sciences, pendant l'année 1821, partie physique. Mémoires de l'Académie des Sciences de l'Institut de France Paris, 5: 176-184.

SzederJesi, T., PAvlíčeK, T., CoşKun, Y. \& Csuzdi, Cs. (2014a): New earthworm records from Turkey, with description of three new species (Oligochaeta: Lumbricidae). Zootaxa, 3764(5): 555-570. doi: $\underline{10.11646 / \text { zootaxa.3764.5.4 }}$

SZEDERJESI, T., PAVlíčEK, T., LATIF, R. \& Csuzdi, Cs. (2014b): Review of the Eisenia muganiensis (Michaelsen, 1910) species group with description of two new species (Oligochaeta: Lumbricidae). Zootaxa, 3884(3): 282-288. doi: $10.11646 /$ zootaxa.3884.3.7 